\title{
A GENERAL EXTREMAL PROBLEM FOR THE CLASS OF CLOSE-TO-CONVEX FUNCTIONS(')
}

\author{
BY
}

JOHN G. MILCETICH

\begin{abstract}
For $\beta \geqslant 0, K_{\beta}$ denotes the set of functions $f(z)=z+a_{2} z^{2}$ $+\cdots$ defined on the unit disc $U$ with the representation $f^{\prime}(z)$ $=a p^{\beta}(z) s(z) / z$, where $a \in C, p$ is an analytic function with positive real part in $U$, and $s$ is a normalized starlike function. If $0 \leqslant \beta \leqslant 1$, and $\zeta \in U$, let $F(u, v)$ be analytic in a neighborhood of $\left\{(f(\zeta), \zeta): f \in K_{\beta}\right\}$. Then $\max \left\{\operatorname{Re} F(f(\zeta), \zeta): f \in K_{\beta}\right\}$ occurs for a function of the form

$$
f(z)=(\beta+1)^{-1}(x-y)^{-1}\left[(1+x z)^{\beta+1}(1+y z)^{-\beta-1}-1\right],
$$

where $|x|=|y|=1$ and $x \neq y$. If $0<\beta<1$ these are the only extremal functions. A consequence of this result is the determination of the value region $\left\{f(\zeta) / \zeta: f \in K_{\beta}\right\}$ as $\left\{(\beta+1)^{-1}(s-t)^{-1}\left[(1+s)^{\beta+1}(1+t)^{-\beta-1}-1\right]\right.$ : $|s|,|t| \leqslant|\zeta|\}$.
\end{abstract}

0 . Introduction. Let $U$ denote the unit disc $\{z:|z|<1\}$ and $S^{*}$ the family of univalent functions $s(z)=z+a_{2} z^{2}+\cdots$ that map $U$ onto a starlike domain. An analytic function $f(z)=z+a_{2} z^{2}+\cdots$ defined on $U$ is said to be closeto-convex of order $\beta, \beta \geqslant 0$, if there exists a complex number $a$, a function $s \in S^{*}$, and a function $p$ analytic and with positive real part in $U$ such that

$$
f^{\prime}(z)=a p^{\beta}(z) s(z) / z .
$$

Since $f^{\prime}(0)=1$, we may always assume that $|a|=|p(0)|=1$. The family of all functions which are close-to-convex of order $\beta$ will be denoted $K_{\beta}$. The class $K_{1}$ was introduced by Kaplan [6] and the general class $K_{\beta}$ was introduced later by Pommerenke [11]. Geometrically the class $K_{\beta}, 0 \leqslant \beta \leqslant 1$, is characterized by the property that the tangent vector to any image curve $f(|z|=r)$ never turns back on itself by an angle greater than $\beta \pi$, i.e.,

$$
\int_{\theta_{1}}^{\theta_{2}} \operatorname{Re}\left\{1+z f^{\prime \prime}(z) / f^{\prime}(z)\right\} d \theta>-\beta \pi
$$

Presented to the Society, January 25, 1976; received by the editors September 22, 1975. AMS (MOS) subject classifications (1970). Primary 30A38; Secondary 30A32.

Key words and phrases. Close-to-convex functions, extremal problems, variational methods.

(') This paper formed a part of the author's dissertation, written under the direction of Professor W. E. Kirwan. 
for $0 \leqslant \theta_{1}<\theta_{2} \leqslant 2 \pi+\theta_{1}$ and $z=r e^{i \theta}$. We consider a general extremal problem for the class $K_{\beta}, 0<\beta \leqslant 1$, which we solve using variational methods. We suppose that a function $F(u, v)$ is given which is analytic in a neighborhood of $\left\{(f(\zeta), \zeta): f \in K_{\beta}\right\}$ where $\zeta$ is a fixed point in $U$. We show that $\max _{f \in K_{\beta}} \operatorname{Re} F(f(\zeta), \zeta)$ is attained for a function of the form

$$
f(z)=\frac{1}{\beta+1} \frac{1}{x-y}\left[\left(\frac{1+x z}{1+y z}\right)^{\beta+1}-1\right]
$$

where $|x|=|y|=1$ and $x \neq y$. Such functions map $U$ onto the complement of a wedge of angular opening $(1-\beta) \pi$.

Kirwan [8] solved the corresponding extremal problem for the class $V_{k}, 2 \leqslant k \leqslant 4$, of functions with boundary rotation at most $k \pi$. The class $V_{k}, k \geqslant 2$, consists of all locally univalent, analytic functions $f(z)=z+a_{2} z^{2}$ $+\cdots$ defined in $U$ which satisfy

$$
\int_{0}^{2 \pi}\left|\operatorname{Re}\left\{1+\frac{r e^{i \theta} f^{\prime \prime}\left(r e^{i \theta}\right)}{f^{\prime}\left(r e^{i \theta}\right)}\right\}\right| d \theta \leqslant k \pi .
$$

This integral measures the total variation of the argument of the tangent vector to $f(|z|=r)$. These functions were introduced by Paatero [10]. The classes $V_{k}$ and $K_{\beta}$ are related by the set inclusion $V_{2 \beta+2} \subset K_{\beta}$ [3].

Kirwan based his proof on a variational formula for functions of bounded boundary rotation given by Schiffer and Tammi [13] and on the Julia variational formula [5]. The proof of the stated result for the class $K_{\beta}, 0<\beta$ $\leqslant 1$, depends on the variational formula for starlike functions given by Hummel [4], the variational formula for functions with positive real part given by Robertson [12], and on the Julia variational formula as applied by Kirwan.

We apply this general theorem to investigate the value region of $f(\zeta) / \zeta$, where $\zeta \in U$ is fixed, $|\zeta|=r$. The case $\beta=0$ was studied by Strohhäcker [14] and the case $\beta=1$ by Biernacki [2]. Denoting this region by $D_{r, \beta}$ we show that

$$
\partial D_{r, \beta} \subset\left\{\frac{1}{\beta+1} \frac{1}{s-t}\left[\left(\frac{1+s}{1+t}\right)^{\beta+1}-1\right]:|s|=|t|=r\right\}
$$

and that

$$
D_{r, \beta}=\left\{\frac{1}{\beta+1} \frac{1}{s-t}\left[\left(\frac{1+s}{1+t}\right)^{\beta+1}-1\right]:|s|,|t| \leqslant r\right\} .
$$

From this result we may infer information about the radius of starlikeness of $K_{\beta}$, which is defined to be the radius of the largest disc centered at 0 such that every function in $K_{\beta}$ is starlike in this disc. Denoting this number by $r_{s}(\beta)$ we 
show that

$$
r_{s}(\beta)=\min \left\{r: \operatorname{Re} \frac{1}{\beta+1} \frac{1}{s-t}\left[\left(\frac{1+s}{1+t}\right)^{\beta+1}-1\right]=0 \text { on }|s|=|t|=r\right\} \text {. }
$$

Lewandowski [9] showed explicitly that $r_{s}(1)=4 \sqrt{2}-5$.

1. The variational formulae and outline of proof. Let $0<\beta \leqslant 1$ and suppose that $F(u, v)$ is analytic in a neighborhood of $\left\{(f(\zeta), \zeta): f \in K_{\beta}\right\}$, where $\zeta$ is a fixed point in $U$. We wish to determine the functions in $K_{\beta}$ which yield

$$
\max _{f \in K_{\beta}} \operatorname{Re} F(f(\zeta), \zeta)
$$

The method of proof involves the use of a number of variational formulae which we now describe.

Since $f \in K_{\beta}$, we have

$$
f^{\prime}(z)=a p^{\beta}(z) s(z) / z,
$$

where $s \in S^{*}, \operatorname{Re} p(z)>0, p(0)=e^{i \gamma}$ and $a=e^{-i \beta \gamma}$. The first step in the proof will be to vary the function $s \in S^{*}$ using the Hummel variation for starlike functions [4]. Hummel showed that given an $s \in S^{*}$, there exists a function $s^{*} \in S^{*}$ of the form

$$
\begin{aligned}
& s^{*}(z)=s(z)+\varepsilon\left(1-\left|z_{0}\right|^{2}\right)\left\{\frac { e ^ { i \alpha } } { z _ { 0 } } \left[\frac{s(z)}{2} \frac{z+z_{0}}{z-z_{0}}+\frac{s(z)}{2}\right.\right. \\
& \left.-A\left(z_{0}\right)\left(\frac{z s^{\prime}(z)}{2} \frac{z+z_{0}}{z-z_{0}}-\frac{z s^{\prime}(z)}{2}+s(z)\right)\right] \\
& \left.+\frac{e^{-i \alpha}}{\bar{z}_{0}}\left[\frac{s(z)}{2} \frac{1+z \bar{z}_{0}}{1-z \bar{z}_{0}}-\frac{s(z)}{2}+\bar{A}\left(z_{0}\right)\left(\frac{z s^{\prime}(z)}{2} \frac{1+z \bar{z}_{0}}{1-z \bar{z}_{0}}-\frac{z s^{\prime}(z)}{2}\right)\right]\right\} \\
& +o(\varepsilon)
\end{aligned}
$$

where $A\left(z_{0}\right)=s\left(z_{0}\right) / z_{0} s^{\prime}\left(z_{0}\right), \varepsilon$ is a positive parameter tending to $0, \alpha$ is an arbitrary real number, and $z_{0}$ is an arbitrary point in the unit disc. Also the estimate for $o(\varepsilon)$ is uniform on compact subsets of $U$. The varied function maps $U$ onto a domain obtained from $s(U)$ by making a small radial displacement of the boundary of $s(U)$.

An application of this variation shows that the function $f$ cannot be extremal for (1.1) unless $s$ maps $U$ onto the complement of a finite set of disjoint rays, i.e., $\operatorname{Re}\left\{z s^{\prime}(z) / s(z)\right\}=0$ on $|z|=1$ except for a finite number of points.

The next part of the proof involves varying the function $p$ by means of a formula established by Robertson [12] for functions which are subordinate to a given function. 
Suppose that $p$ is subordinate to a univalent function $p_{0}$ in $U$, i.e., $p(z)=p_{0}(\omega(z))$ where $\omega: U \rightarrow U$ and $\omega(0)=0$. Then

$$
\omega(z)=(Q(z)-1) /(Q(z)+1),
$$

where $\operatorname{Re} Q(z)>0$ for $z \in U$. Robertson gave the following variational formula for $p(z)$ which yields a function $p^{*}$ which is also subordinate to $p_{0}$ :

$$
p^{*}(z)=p(z)-\rho^{2}\left(1-\left|z_{0}\right|^{2}\right) \frac{(1-\phi(p(z)))^{2}}{2 \phi^{\prime}(p(z))} B(z)+o\left(\rho^{2}\right)
$$

where

$$
\begin{aligned}
B(z)= & \frac{2 \phi^{\prime}(p(z)) p^{\prime}(z)}{(1-\phi(p(z)))^{2}}\left\{\frac{e^{i \theta} z}{Q\left(z_{0}\right)\left(z_{0}-z\right)}+\frac{z^{2} e^{-i \theta}}{\overline{Q\left(z_{0}\right)\left(1-\bar{z}_{0} z\right)}}\right\} \\
& +Q(z)\left\{\frac{e^{i \theta} z}{Q\left(z_{0}\right)\left(z_{0}-z\right)^{2}}+\frac{e^{-i \theta} z}{\overline{Q\left(z_{0}\right)}\left(1-\bar{z}_{0} z\right)^{2}}\right\} \\
& +\left\{\frac{e^{-i \theta} z}{\left(1-\bar{z}_{0} z\right)^{2}}-\frac{e^{i \theta} z^{2}}{z_{0}\left(z_{0}-z\right)^{2}}-\frac{e^{i \theta} z}{z_{0}\left(z_{0}-z\right)}\right\},
\end{aligned}
$$

$\phi=p_{0}^{-1}, \theta$ is an arbitrary real number, $z_{0}$ is an arbitrary point of $U, \rho$ is a parameter tending to 0 and the estimate for $o\left(\rho^{2}\right)$ is uniform on compact subsets of $U$.

Using this variation we show that $f$ cannot be extremal for (1.1) unless $\operatorname{Re} p(z)=0$ on $|z|=1$ except for a finite number of points. A calculation then yields that an extremal $f$ must map $U$ onto a polygonal domain. To finish the proof we employ the Julia variational formula as done in [8] in order to show that the extremal domain is the complement of an infinite wedge of opening $(1-\beta) \pi$.

The form of the Julia variation required for our application is that

$$
f^{*}(z)=f(z)+\frac{\varepsilon z f^{\prime}(z)}{2 \pi i} \int_{\Gamma} \frac{\xi+z}{\xi-z} \operatorname{Im}\left\{-\psi(w) \frac{|d w|}{d w}\right\} \frac{n(w)}{\xi^{2} f^{\prime 2}(\xi)} d w+o(\varepsilon)
$$

Here $f(z)=z+a_{2} z+\cdots$ maps $U$ conformally onto the domain with boundary $\Gamma=\{w=f(\xi):|\xi|=1\}, \psi(w)$ is continuous and piecewise differentiable on $\Gamma, n(w)$ is the unit exterior normal to $\Gamma$ at $w$ and $f^{*}(z)=a_{1}^{*} z$ $+a_{2}^{*} z+\cdots$ maps $U$ conformally onto the domain $D^{*}$ with boundary $\Gamma^{*}=\left\{w^{*}=w+\varepsilon \psi(w)\right\}$ where $\varepsilon>0$.

We now outline the method of application of the Julia variation which we will use. This method was originated by Biernacki [2] and has been refined by others (e.g., [1], [8]). Let $\zeta$ be fixed, $|\zeta|<1$. Let $f \in K_{\beta}$ and let $f^{*}$ be defined by (1.5). We expand $F\left(f^{*}(\zeta), \zeta\right)$ in powers of $\varepsilon$, obtaining 


$$
\begin{aligned}
& F\left(f^{*}(\zeta), \zeta\right)=F(f(\zeta), \zeta) \\
& +\varepsilon \zeta f^{\prime}(\zeta) \frac{F_{1}(f(\zeta), \zeta)}{2 \pi i} \int_{\Gamma} \frac{z+\zeta}{z-\zeta} \operatorname{Im}\left\{-\psi(w) \frac{|d w|}{d w}\right\} \frac{n(w)}{z f^{\prime 2}(z)} d w+o(\varepsilon)
\end{aligned}
$$

where we have set $F_{1}(u, v)=\partial F(u, v) / \partial u$.

Let us assume that the boundary of $f(U)$ contains three disjoint analytic arcs, which then correspond to three arcs $l_{1}, l_{2}$, and $l_{3}$ on $|z|=1$. For fixed $\zeta, \zeta f^{\prime}(\zeta) F_{1}(f(\zeta), \zeta)(z+\zeta) /(z-\zeta)$ traces out a circle as $z$ varies on $|z|=1$. Hence, among the three arcs $l_{1}, l_{2}$, and $l_{3}$ there are two, which we denote by $\gamma_{1}$ and $\gamma_{2}$, satisfying

$$
\begin{aligned}
\max _{z \in \gamma_{1}} & \operatorname{Re}\left\{\zeta f^{\prime}(\zeta) F_{1}(f(\zeta), \zeta)(z+\zeta) /(z-\zeta)\right\} \\
& <\min _{z \in \gamma_{2}} \operatorname{Re}\left\{\zeta f^{\prime}(\zeta) F_{1}(f(\zeta), \zeta)(z+\zeta) /(z-\zeta)\right\}
\end{aligned}
$$

Let us choose $\psi(w)$ so that

$$
\operatorname{Im}\left\{-\psi(w) \frac{|d w|}{d w}\right\} \begin{cases}<0 & \text { if } w \in f\left(\gamma_{1}\right) \\ >0 & \text { if } w \in f\left(\gamma_{2}\right) \\ =0 & \text { if } w \in \Gamma \backslash\left(f\left(\gamma_{1}\right) \cup f\left(\gamma_{2}\right)\right)\end{cases}
$$

and

$$
\int_{\Gamma} \operatorname{Im}\left\{-\psi(w) \frac{|d w|}{d w}\right\} \frac{n(w)}{z^{2} f^{\prime 2}(z)} d w=0 .
$$

An application of the variational formula (1.6) to $f$ with this choice of $\psi(w)$ yields $f^{* \prime}(0)=1$ by $(1.9)$ and

$$
\operatorname{Re}\left\{\zeta f^{\prime}(\zeta) \frac{F_{1}(f(\zeta), \zeta)}{2 \pi i} \int_{\Gamma} \frac{z+\zeta}{z-\zeta} \operatorname{Im}\left\{-\psi(w) \frac{|d w|}{d w}\right\} \frac{n(w)}{z f^{\prime 2}(z)} d w\right\}>0
$$

by (1.7), (1.8) and (1.9). Hence, from (1.6) we conclude that

$$
\operatorname{Re} F\left(f^{*}(\zeta), \zeta\right)>\operatorname{Re} F(f(\zeta), \zeta)
$$

and, consequently, if $f^{*} \in K_{\beta}$, then $f$ is not extremal for problem (1.1). The following lemma summarizes the above discussion.

LEMMA 1.1. Let $f \in K_{\beta}, 0<\beta<1$, and suppose that the boundary of $f(U)$ contains three disjoint analytic arcs. Moreover let $F(u, v)$ be analytic in $\bigcup_{f \in K_{\beta}}(f(\zeta), \zeta)$ where $\zeta$ is fixed, $|\zeta|<1$. Then

(a) there exist two arcs $\gamma_{1}$ and $\gamma_{2}$ on $\partial U$ that satisfy (1.7);

(b) in addition, if $\psi(w)$ is defined on $\partial f(U)$ to satisfy (1.8) and (1.9) and if $f^{*}$ 
defined by (1.5) belongs to $K_{\beta}$, then $f$ is not an extremal function for problem (1.1).

2. Statement and proof of the main theorem. We are now ready to state the main theorem.

THEOREM 2.1. Let $\zeta$ be a fixed point of $U$ and suppose that $F(u, v)$ is analytic in a neighborhood of $\left\{(f(\zeta), \zeta): f \in K_{\beta}\right\}$ where $0<\beta \leqslant 1$. Then $\max _{f \in K_{\beta}} \operatorname{Re} F(f(\zeta), \zeta)$ is assumed for a function of the form

$$
f(z)=\frac{1}{\beta+1} \frac{1}{x-y}\left[\left(\frac{1+x z}{1+y z}\right)^{\beta+1}-1\right]
$$

where $|x|=|y|=1$ and $x \neq y$. If $0<\beta<1$ and if $F$ is nonconstant, functions of the form (2.1) are the only functions for which the maximum is attained.

We remark that a function of the form (2.1) maps $U$ onto the complement of a wedge with angular opening $(1-\beta) \pi$. We also note that the case $\beta=0$ was established by Kirwan [8].

Before beginning the proof of the theorem, it is necessary to prove the following lemma.

LEMMA 2.2. Let $f \in K_{\beta}$ be an extremal function for (1.1) and suppose that $f^{\prime}(z)=a p(z)^{\beta} s(z) / z$ where $|a|=1, a \cdot p(0)=1, \operatorname{Re} p(z)>0$ for $|z|<1$ and $s \in S^{*}$. Then

$$
\operatorname{Im}\left\{\frac{\partial F}{\partial u}(f(\zeta), \zeta)\left[f(\zeta)-\int_{0}^{\zeta} a p^{\beta}(z) s^{\prime}(z) d z\right]\right\}=0
$$

and

$$
\operatorname{Im}\left\{\frac{\partial F}{\partial u}(f(\zeta), \zeta) \cdot \int_{0}^{\zeta} a p^{\beta-1}(z) s(z) p^{\prime}(z) d z\right\}=0
$$

Proof. We have

$$
f(\xi)=\int_{0}^{\xi} a p^{\beta}(z) \frac{s(z)}{z} d z .
$$

If $\tau \in \mathbf{R}$ the function $e^{i \tau} s\left(e^{-i \tau} z\right)$ is also in $S^{*}$ and we define the function $f_{\tau} \in K_{\beta}$ by

$$
f_{\tau}(\xi)=\int_{0}^{\xi} a p^{\beta}(z) e^{i \tau} \frac{s\left(e^{-i \tau} z\right)}{z} d z .
$$

But

$$
s\left(e^{-i \tau} z\right)=s(z-i \tau z+o(\tau))=s(z)-s^{\prime}(z) i \tau z+o(\tau)
$$


and $e^{i \tau}=1+i \tau+o(\tau)$. Therefore

$$
\begin{aligned}
f_{\tau}(\xi) & =\int_{0}^{\xi} a p^{\beta}(z)\left\{\frac{s(z)+i \tau\left[s(z)-z s^{\prime}(z)\right]}{z}+o(\tau)\right\} d z \\
& =f(\xi)+i \tau\left[f(\xi)-\int_{0}^{\xi} a p^{\beta}(z) s^{\prime}(z) d z\right]+o(\tau) .
\end{aligned}
$$

In these formulae, the $o(\tau)$-estimate is uniform on compact subsets of $U$. Expanding $F\left(f_{\tau}(\zeta), \zeta\right)$ in powers of $\tau$ and recalling that $f$ is an extremal function, we obtain

$\operatorname{Re} F\left(f_{\tau}(\zeta), \zeta\right)$

$$
\begin{aligned}
& =\operatorname{Re} F(f(\zeta), \zeta)+\operatorname{Re}\left\{i \tau F_{1} \cdot\left[f(\zeta)-\int_{0}^{\zeta} a p^{\beta}(z) s^{\prime}(z) d z\right]\right\}+o(\tau) \\
& \leqslant \operatorname{Re} F(f(\zeta), \zeta),
\end{aligned}
$$

where we have set $F_{1}=\partial F(f(\zeta), \zeta) / \partial u$. Hence

$$
\operatorname{Re}\left\{i \tau F_{1} \cdot\left[f(\zeta)-\int_{0}^{\zeta} a p^{\beta}(z) s^{\prime}(z) d z\right]\right\}+o(\tau) \leqslant 0 .
$$

Since $\tau$ can take on either positive or negative values, we conclude that

$$
\begin{aligned}
0 & =\operatorname{Re}\left\{i F_{1} \cdot\left[f(\zeta)-\int_{0}^{\zeta} a p^{\beta}(z) s^{\prime}(z) d z\right]\right\} \\
& =-\operatorname{Im}\left\{F_{1} \cdot\left[f(\zeta)-\int_{0}^{\zeta} a p^{\beta}(z) d z\right]\right\}
\end{aligned}
$$

which is (2.2).

To obtain the second relation, we set

$$
f_{\tau}(\xi)=\int_{0}^{\xi} a p^{\beta}\left(e^{-i \tau} z\right) \frac{s(z)}{z} d z,
$$

where $\tau \in \mathbf{R}$. Then $f_{\tau} \in K_{\beta}$. Moreover,

$$
p\left(e^{-i \tau} z\right)=p(z-i \tau z+o(\tau))=p(z)-i \tau z p^{\prime}(z)+o(\tau)
$$

and, hence,

$$
p^{\beta}\left(e^{-i \tau} z\right)=p^{\beta}(z)-\beta i \tau z p^{\prime}(z) p^{\beta-1}(z)+o(\tau) .
$$

Here again the estimate $o(\tau)$ is uniform on compact subsets of $U$. Then 


$$
\begin{aligned}
f_{\tau}(\xi) & =\int_{0}^{\xi} a\left[p^{\beta}(z)-\beta i \tau z p^{\prime}(z) p^{\beta-1}(z)\right] \frac{s(z)}{z} d z+o(\tau) \\
& =f(\xi)-\beta i \tau \int_{0}^{\xi} a p^{\prime}(z) p^{\beta-1}(z) s(z) d z+o(\tau) .
\end{aligned}
$$

We again expand $F\left(f_{\tau}(\zeta), \zeta\right)$ in powers of $\tau$ and obtain

$\operatorname{Re} F\left(f_{\tau}(\zeta), \zeta\right)$

$$
\begin{aligned}
& =\operatorname{Re} F(f(\zeta), \zeta)+\operatorname{Re} F_{1} \cdot\left[-\beta i \tau \int_{0}^{\zeta} a p^{\beta-1}(z) p^{\prime}(z) s(z) d z\right]+o(\tau) \\
& \leqslant \operatorname{Re} F(f(\zeta), \zeta) .
\end{aligned}
$$

Since $f$ is assumed to be an extremal function and since $\tau$ may assume both positive and negative values, it follows that

$$
\operatorname{Re} F_{1} \cdot i \int_{0}^{\zeta} a p^{\beta-1}(z) p^{\prime}(z) s(z) d z=0
$$

i.e.,

$$
\operatorname{Im} F_{1} \cdot \int_{0}^{\zeta} a p^{\beta-1}(z) p^{\prime}(z) s(z) d z=0
$$

which is the second relation.

Proof of theOREM. Let $f \in K_{\beta}$ be an extremal function for (1.1). Then $f^{\prime}(z)=a p^{\beta}(z) s(z) / z$ where $|a|=|p(0)|=1, \operatorname{Re} p(z)>0$ and $s \in S^{*}$. Our first aim is to show that $s(z)$ has an analytic extension to $|z|=1$ with a finite number of points deleted and that, except for these exceptional points, $\operatorname{Re}\left\{z s^{\prime}(z) / s(z)\right\}=0$ on $|z|=1$.

We apply the Hummel variation to $s$ obtaining a function $s^{*} \in S^{*}$ and we set $f^{* \prime}(z)=a p^{\beta}(z) s^{*}(z) / z$. Then

$$
f^{*}(\zeta)=\int_{0}^{\zeta} a p^{\beta}(z) \frac{s^{*}(z)}{z} d z
$$

and expanding $F\left(f^{*}(\zeta), \zeta\right)$ in powers of $\varepsilon$ with the aid of (1.3), we obtain

$$
F\left(f^{*}(\zeta), \zeta\right)=F(f(\zeta), \zeta)+\partial F(f(\zeta), \zeta) / \partial u \cdot h\left(z_{0}\right)+o(\varepsilon)
$$

where

$$
\begin{aligned}
h\left(z_{0}\right)=\varepsilon\left(1-\left|z_{0}\right|^{2}\right) \int_{0}^{\zeta} \frac{a p^{\beta}(z)}{z}\{ & \frac{e^{i \alpha}}{z_{0}}\left[\frac{s(z)}{2} \frac{z+z_{0}}{z-z_{0}}+\frac{s(z)}{2}\right. \\
& \left.-A\left(z_{0}\right)\left(\frac{z s^{\prime}(z)}{2} \frac{z+z_{0}}{z-z_{0}}-\frac{z s^{\prime}(z)}{2}+s(z)\right)\right] \\
+ & \frac{e^{-i \alpha}}{\bar{z}_{0}}\left[\frac{s(z)}{2} \frac{1+\bar{z}_{0} z}{1-\bar{z}_{0} z}-\frac{s(z)}{2}+\bar{A}\left(z_{0}\right)\right. \\
& \left.\left.\cdot\left(\frac{z s^{\prime}(z)}{2} \frac{1+\bar{z}_{0} z}{1-\bar{z}_{0} z}-\frac{z s^{\prime}(z)}{2}\right)\right]\right\} d z .
\end{aligned}
$$


Since $f$ is an extremal function for (1.1),

$$
\operatorname{Re} F\left(f^{*}(\zeta), \zeta\right) \leqslant \operatorname{Re} F(f(\zeta), \zeta)
$$

From (2.4) we obtain

$$
\operatorname{Re} F_{1} \cdot h\left(z_{0}\right)+o(\varepsilon) \leqslant 0,
$$

where we have set $F_{1}=\partial F(f(\zeta), \zeta) / \partial u$. Dividing by $\varepsilon\left(1-\left|z_{0}\right|^{2}\right)$ and using the fact that $\operatorname{Re}\{a M+\bar{a} N\}=\operatorname{Re}\{a(M+\bar{N})\}$, we have

$$
\begin{aligned}
0 \geqslant & \operatorname{Re} e^{i \alpha}\left\{F _ { 1 } \cdot \int _ { 0 } ^ { \zeta } a \frac { p ^ { \beta } ( z ) } { z } \frac { 1 } { z _ { 0 } } \left[\frac{s(z)}{2} \frac{z+z_{0}}{z-z_{0}}+\frac{s(z)}{2}\right.\right. \\
& \left.-A\left(z_{0}\right)\left(\frac{z s^{\prime}(z)}{2} \frac{z+z_{0}}{z-z_{0}}-\frac{z s^{\prime}(z)}{2}+s(z)\right)\right] d z \\
+ & \frac{F_{1}}{\left.\int_{0}^{\zeta} a p^{\beta}(z) \frac{1}{z_{0}}\left[\frac{s(z)}{2} \frac{1+\bar{z}_{0} z}{1-\bar{z}_{0} z}-\frac{s(z)}{2}+\bar{A}\left(z_{0}\right)\left(\frac{z s^{\prime}(z)}{2} \frac{1+z \bar{z}_{0}}{1-\bar{z}_{0} z}-\frac{z s^{\prime}(z)}{2}\right)\right] d z\right\} .}
\end{aligned}
$$

But $\alpha$ is an arbitrary real number so we conclude that

$$
\begin{aligned}
& 0=F_{1} \int_{0}^{\zeta} \frac{a p^{\beta}(z)}{z} \frac{1}{z_{0}}\left(\frac{s(z)}{2} \frac{z+z_{0}}{z-z_{0}}+\frac{s(z)}{2}\right. \\
& \left.-A\left(z_{0}\right)\left[\frac{z s^{\prime}(z)}{2} \frac{z+z_{0}}{z-z_{0}}-\frac{z s^{\prime}(z)}{2}+s(z)\right]\right) d z
\end{aligned}
$$

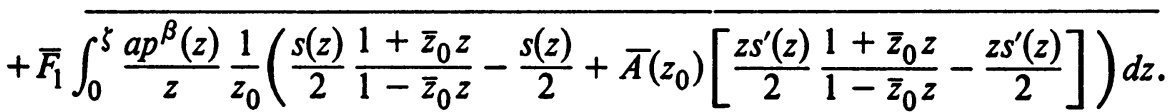

The solution of the above equation for $A\left(z_{0}\right)=s\left(z_{0}\right) / z_{0} s^{\prime}\left(z_{0}\right)$ yields

$$
z_{0} s^{\prime}\left(z_{0}\right) / s\left(z_{0}\right)=Q\left(z_{0}\right) / R\left(z_{0}\right)
$$

where

$$
\begin{aligned}
Q\left(z_{0}\right)= & F_{1} \int_{0}^{\zeta} \frac{a p^{\beta}(z)}{z}\left(\frac{z s^{\prime}(z)}{2} \frac{z+z_{0}}{z-z_{0}}-\frac{z s^{\prime}(z)}{2}+s(z)\right) d z \\
& -\bar{F}_{1} \overline{\int_{0}^{\zeta}\left(\frac{z s^{\prime}(z)}{2} \frac{1+\bar{z}_{0} z}{1-\bar{z}_{0} z}-\frac{z s^{\prime}(z)}{2}\right) \frac{a p^{\beta}(z)}{z} d z}
\end{aligned}
$$

and

$$
\begin{aligned}
R\left(z_{0}\right)= & F_{1} \int_{0}^{\zeta} \frac{a p^{\beta}(z)}{z}\left(\frac{s(z)}{2} \frac{z+z_{0}}{z-z_{0}}+\frac{s(z)}{2}\right) d z \\
& +\bar{F}_{1} \overline{\int_{0}^{\zeta} \frac{a p^{\beta}(z)}{z}\left(\frac{s(z)}{2} \frac{1+\bar{z}_{0} z}{1-\bar{z}_{0} z}-\frac{s(z)}{2}\right) d z .}
\end{aligned}
$$


From their definitions it is clear that $Q\left(z_{0}\right)$ and $R\left(z_{0}\right)$ are not identically zero unless $F_{1}=0$. But by a result in [7], this can only occur if $F$ is identically constant, in which case the theorem is trivial. The equation $z_{0} s^{\prime}\left(z_{0}\right) / s\left(z_{0}\right)$ $=Q\left(z_{0}\right) / R\left(z_{0}\right)$ holds for each $z_{0} \in U$. Let us replace $z_{0}$ by $t$. We see that $Q(t)$ and $R(t)$ are defined in the annulus $|\zeta|<|t|<1 /|\zeta|$ and, hence, $s$ has an analytic extension to $|t|=1$, except possibly at the finite number of zeros of $R$ on $|t|=1$. A simple calculation shows that if $|t|=1$ then $R(t)=\overline{-R(1 / t)}$ and

$$
\begin{aligned}
\frac{Q(t)}{R(t)}+\frac{\overline{Q(1 / \bar{t})}}{\overline{R(1 / \bar{t})}}= & \frac{1}{R(t)}\left\{F_{1} \int_{0}^{\zeta} a p^{\beta}(z)\left[\frac{s(z)}{z}-s^{\prime}(z)\right] d z\right. \\
& \left.-\bar{F}_{1} \overline{\int_{0}^{\zeta} a p^{\beta}(z)\left[\frac{s(z)}{z}-s^{\prime}(z)\right] d z}\right\} \\
= & 2 i \operatorname{Im} F_{1}\left\{f(\zeta)-\int_{0}^{\zeta} a p^{\beta}(z) s^{\prime}(z) d z\right\} / R(t) \\
= & 0
\end{aligned}
$$

by (2.2). Therefore

$$
\operatorname{Re}\left\{t s^{\prime}(t) / s(t)\right\}=0 \text { on }|t|=1
$$

except for a finite number of points. This implies that $\arg s\left(e^{i \theta}\right)$ is constant on a finite number of intervals of $|t|=1$ whose closure is $|t|=1$.

We now assume that the extremal $f \in K_{\beta}$ satisfies $f^{\prime}(z)=a p(z) s(z) / z$, where $s \in S^{*}$ satisfies (2.5). Our aim is to show that $p(z)$ has an analytic extension to $|z|=1$ with a finite number of points deleted and that except for these exceptional points, $\operatorname{Re} p(z)=0$ on $|z|=1$. We let $p(0)=e^{i \gamma}$ where $-\pi / 2<\gamma<\pi / 2$. Then $p$ is subordinate to $p_{\gamma}(z)=\left(e^{i \gamma}+z\right) /\left(1-e^{i \gamma} z\right)$ and we will now vary $p$ using (1.4). Then

$$
p^{* \beta}(z)=p^{\beta}(z)+p^{\beta-1}(z) \cdot \beta\left(p^{*}(z)-p(z)\right)+o\left(\rho^{2}\right)
$$

and

$$
f^{*}(\zeta)=f(\zeta)+\int_{0}^{\zeta} \frac{k(z)}{z}\left(p^{*}(z)-p(z)\right) d z+o\left(\rho^{2}\right)
$$

where $k(z)=a \beta p^{\beta-1}(z) s(z)$. Expanding $F\left(f^{*}(\zeta), \zeta\right)$ in powers of $\rho^{2}$ and again setting $F_{1}=\partial F(f(\zeta), \zeta) / \partial u$, we obtain

$$
\operatorname{Re} F\left(f^{*}(\zeta), \zeta\right)=\operatorname{Re} F(f(\zeta), \zeta)+\operatorname{Re} F_{1} \cdot \int_{0}^{\zeta} \frac{k(z)}{z}\left(p^{*}(z)-p(z)\right) d z+o\left(\rho^{2}\right)
$$

and since $f$ is extremal, 


$$
\operatorname{Re} F_{1} \cdot \int_{0}^{\zeta} \frac{k(z)}{z}\left(p^{*}(z)-p(z)\right) d z+o\left(\rho^{2}\right) \leqslant 0 .
$$

If we set $l(z)=(1-\phi(p(z)))^{2} / 2 \phi^{\prime}(p(z))$, divide both sides of the above inequality by $-\rho^{2}\left(1-\left|z_{0}\right|^{2}\right)$, and let $\rho$ tend to 0 , we get

$$
\begin{aligned}
0 \leqslant \operatorname{Re}\left[F _ { 1 } e ^ { i \theta } \int _ { 0 } ^ { \zeta } k ( z ) \left\{\frac{p^{\prime}(z)}{Q\left(z_{0}\right)\left(z_{0}-z\right)}+\frac{Q(z) l(z)}{Q\left(z_{0}\right)\left(z_{0}-z\right)^{2}}\right.\right. \\
\left.-\left[\frac{z l(z)}{z_{0}\left(z_{0}-z\right)^{2}}+\frac{l(z)}{z_{0}\left(z_{0}-z\right)}\right]\right\} d z \\
+F_{1} e^{-i \theta} \int_{0}^{\zeta} k(z)\left\{\frac{p^{\prime}(z) z}{\overline{Q\left(z_{0}\right)}\left(1-\bar{z}_{0} z\right)}\right. \\
\left.\left.+\frac{Q(z) l(z)}{\overline{Q\left(z_{0}\right)}\left(1-\bar{z}_{0} z\right)^{2}}+\frac{l(z)}{\left(1-\bar{z}_{0} z\right)^{2}}\right\} d z\right] .
\end{aligned}
$$

Since $\operatorname{Re}\{a M+\bar{a} N\}=\operatorname{Re}\{a(M+\bar{N})\}$ and since $\theta$ is arbitrary, we find that

$$
Q\left(z_{0}\right)=A\left(z_{0}\right) / B\left(z_{0}\right)
$$

where

$$
\begin{aligned}
A\left(z_{0}\right)= & F_{1} \int_{0}^{\zeta}\left(\frac{k(z) p^{\prime}(z)}{\left(z_{0}-z\right)}+\frac{k(z) Q(z) l(z)}{\left(z_{0}-z\right)^{2}}\right) d z \\
& +F_{1} \int_{0}^{\zeta}\left(\frac{z k(z) p^{\prime}(z)}{1-\bar{z}_{0} z}+\frac{k(z) l(z) Q(z)}{\left(1-\bar{z}_{0} z\right)^{2}}\right) d z
\end{aligned}
$$

and

$$
B\left(z_{0}\right)=F_{1} \int_{0}^{\zeta} \frac{k(z) l(z)}{\left(z_{0}-z\right)^{2}} d z-F_{1} \overline{\int_{0}^{\zeta} \frac{k(z) l(z)}{\left(1-\bar{z}_{0} z\right)^{2}} d z .}
$$

The relation $Q\left(z_{0}\right)=A\left(z_{0}\right) / B\left(z_{0}\right)$ holds for every $z_{0} \in U$. As before, we replace $z_{0}$ by $t$. We see that $A(t)$ and $B(t)$ are not identically zero since we can assume $F_{1} \neq 0$ and also that $A$ and $B$ are defined in the annulus $|\zeta|<|t|$ $<1 /|\zeta|$. Calculating $\overline{Q(1 / \bar{t})}$, we obtain

$$
\begin{aligned}
\overline{Q(1 / \bar{t})}= & \left(F_{1} \int_{0}^{\zeta}\left(\frac{z k(z) p^{\prime}(z) t}{t-z}+\frac{k(z) l(z) Q(z) z_{0}^{2}}{(t-z)^{2}}\right) d z\right. \\
& \left.+\bar{F}_{1} \overline{\int_{0}^{\zeta}\left(\frac{k(z) p^{\prime}(z) \bar{z}_{0}}{1-\bar{t} z}+\frac{k(z) Q(z) l(z) \bar{t}^{2}}{(1-\bar{t} z)^{2}}\right) d z}\right) /-t^{2} B(t)
\end{aligned}
$$

and hence 


$$
\begin{aligned}
Q(t)+\overline{Q(1 / \bar{t})} & =\left(F_{1} \cdot \int_{0}^{\zeta} k(z) p^{\prime}(z) d z-\bar{F}_{1} \overline{\int_{0}^{\zeta} k(z) p^{\prime}(z) d z}\right) / t B(t) \\
& =\left(2 i \operatorname{Im} F_{1} \int_{0}^{\zeta} k(z) p^{\prime}(z) d z\right) / t B(t)=0
\end{aligned}
$$

by (2.3). If $|t|=1$, then $\operatorname{Re} Q(t)=0$ except for the finite number of zeros of $B$ on $|t|=1$. Since $Q$ is defined on $|t| \leqslant 1$ and $Q(t)=-\overline{Q(1 / \bar{t})}$ on $|t|=1, Q$ is meromorphic on $\mathbf{C}$. Then $\omega(t)=(Q(t)-1) /(Q(t)+1)$ is also meromorphic on $\mathbf{C}$ and

$$
\omega(1 / \bar{t})=\frac{Q(1 / \bar{t})-1}{Q(1 / \bar{t})+1}=\frac{\overline{Q(t)}+1}{\overline{Q(t)}-1}=\frac{1}{\omega(t)} .
$$

This implies that $|\omega(t)|=1$ if $|t|=1$. Since $p(t)=p_{\gamma}(\omega(t))$, $p$ has a meromorphic extension to $\mathbf{C}$ and

$$
p(1 / \bar{t})=p_{\gamma}(\omega(1 / \bar{t}))=p_{\gamma}(1 / \overline{\omega(t)})=\overline{-p_{\gamma}(\omega(t))}=\overline{-p(t)}
$$

Thus except for a finite number of points on $|t|=1, \operatorname{Re} p(t)=0$. We observe that for $z=r e^{i \theta}$,

$$
\operatorname{Re}\left\{z p^{\prime}(z) / p(z)\right\}=(\partial / \partial \theta) \arg p\left(r e^{i \theta}\right),
$$

and, hence, except for a finite number of points on $|t|=1, \operatorname{Re}\left\{t p^{\prime}(t) / p(t)\right\}$ $=0$.

We see that the extremal function $f$ of (1.1) has an analytic extension to $|z|=1$ with a finite number of points deleted. We now show that $f$ maps $U$ onto a polygonal domain. Writing $f^{\prime}(z)=a p(z)^{\beta} s(z) / z$ and using the above observation together with (2.5), we compute

$$
\operatorname{Re}\left\{1+\frac{z f^{\prime \prime}(z)}{f^{\prime}(z)}\right\}=\operatorname{Re}\left\{\beta z \frac{p^{\prime}(z)}{p(z)}+\frac{z s^{\prime}(z)}{s(z)}\right\}=0
$$

for all but finitely many $z$ with $|z|=1$. But $\operatorname{Re}\left\{1+z f^{\prime \prime}(z) / f^{\prime}(z)\right\}$ for $|z|=r$ measures the rate of turn of the tangent vector to the curve $f(\{|z|=r\})$. Since $f$ is piecewise analytic on $\{|z|=1\}$ we see that the boundary of $f(U)$ is composed of a finite number of line segments, rays, or lines. Hence $f$ maps $U$ onto a polygonal domain.

We now show that the extremal polygon has only one finite vertex and that the exterior angular opening is $(1-\beta) \pi$. For this we need the following geometric characterization due to Pommerenke [11]. Let $f(z)=z+a_{2} z^{2}$ $+\cdots$ be a conformal map of $U$. Then $f \in K_{\beta}, 0 \leqslant \beta \leqslant 1$, if and only if the following condition holds:

(C) The complement, $E$, of $f(U)$ is the union of rays which are disjoint (except that perhaps the origin of one ray may lie on another ray) and which 
have the property that for each ray in $E$ the sector of opening $(1-\beta) \pi$ whose bisector is the given ray lies in $E$.

Suppose $f$ is a polygonal mapping and $f(U)$ is not a wedge of exterior angular opening $(1-\beta) \pi$. Kirwan [8] has constructed a variation for polygonal domains which preserves the angle at each vertex. One easily checks, using condition (C), that if a polygonal domain is the image of a function $f$ of class $K_{\beta}$, then the polygon obtained by this method of variation is also the image of a function in $K_{\beta}$. Hence we may define a function $\psi$ on $\partial f(U)$ so that the hypotheses of Lemma 1.1 are satisfied and we conclude that $f$ is not extremal for problem (1.1). Consequently $f$ maps $U$ onto a wedge of exterior angular opening $(1-\beta) \pi$ and therefore $f$ has the form (2.1) where $|x|=|y|$ $=1$ and $x \neq y$. This completes the proof of the theorem in the case $0<\beta$ $<1$.

Let $\beta=1$ and let $f \in K_{1}$ be extremal for (1.1). For $0<\gamma<1$, we claim $f_{\gamma}(z)=f(\gamma z) / \gamma \in K_{\beta(\gamma)}$ with $\beta(\gamma)<1$. To see this, write $f^{\prime}(z)=a p(z)^{\beta} s(z) / z$ as in (1.2). Then $f_{\gamma}^{\prime}(z)=a p(\gamma z)^{\beta} s(\gamma z) / \gamma z$. Now $s_{\gamma}(z)=s(\gamma z) / \gamma \in S^{*}$ and $|\arg p(\gamma z)| \leqslant A_{\gamma} \pi / 2<\pi / 2$. If we set $p_{\gamma}(z)=p(\gamma z)^{\gamma_{1} / A_{\gamma}}$, then $p_{\gamma}$ has positive real part in $U$ and $f_{\gamma}^{\prime}(z)=a p_{\gamma}(z)^{\beta A_{\gamma}} s_{\gamma}(z) / z$, showing that $f_{\gamma} \in K_{\beta A_{\gamma}}$ where $\beta A_{\gamma}<1$. Applying the theorem, in the case $\beta=\beta(\gamma)$, there is a function $g_{\gamma}$ of the form (2.1) such that $\operatorname{Re} F\left(f_{\gamma}(\zeta), \zeta\right) \leqslant \operatorname{Re} F\left(g_{\gamma}(\zeta), \zeta\right)$. For some increasing sequence $\left\{\gamma_{n}\right\}$ tending to $1,\left\{g_{\gamma_{n}}\right\}$ converges to a limit function $g$ in $K_{1}$, which is again of the form $(2.1)$, with $\beta \leqslant 1$. Hence $\operatorname{Re} F(f(\zeta), \zeta)$ $\leqslant \operatorname{Re} F(g(\zeta), \zeta)$ and since $f$ was assumed to be extremal, the inequality is in fact an equality. It remains only to show that $g$ is of the form (2.1) with $\beta=1$. If not, $g \in K_{\beta}, \beta<1$, and hence could not be extremal for (1.1) in $K_{\beta^{\prime}}, \beta<\beta^{\prime}<1$. This completes the proof of Theorem 2.1.

3. Applications. Let $0<\beta<1$ and let $\zeta$ be fixed, $|\zeta|=r$. We wish to investigate the value region of $f(\zeta) / \zeta$ as $f$ varies through $K_{\beta}$. The case $\beta=1$ was studied by Biernacki [2] and the case $\beta=0$ by Strohhäcker [14]. We define

$$
D_{r, \beta}=\left\{f(\zeta) / \zeta: f \in K_{\beta}\right\}
$$

and let $\partial D_{r, \beta}$ denote the boundary of $D_{r, \beta}$.

THEOREM 3.1. If $|\xi|=r<1$, then

$$
\begin{aligned}
\partial D_{r, \beta} & \subset\left\{\frac{1}{\beta+1} \frac{1}{s-t}\left[\left(\frac{1+s}{1+t}\right)^{\beta+1}-1\right]:|s|=|t|=r\right\} \\
D_{r, \beta} & =\left\{\frac{1}{\beta+1} \frac{1}{s-t}\left[\left(\frac{1+s}{1+t}\right)^{\beta+1}-1\right]:|s|,|t| \leqslant r\right\} .
\end{aligned}
$$


Proof. (a) We first show that there exists a set $E \subset D_{r, \beta}$ which is everywhere dense in $\partial D_{r, \beta}$ and has the property that if $u_{1} \in E$ there is a point $v \in C D_{r, \beta}$ such that $\left\{u:|u-v|<\left|u_{1}-v\right|\right\} \subset e D_{r, \beta}$. To see this, let $w$ be an arbitrary point of $\partial D_{r, \beta}$ and let $\varepsilon>0$. Choose $v \in e_{r, \beta}$ such that $|w-v|$ $<\varepsilon / 2$. The family $K_{\beta}$ is compact so $D_{r, \beta}$ is closed and hence there is $\delta>0$ such that $\{u:|u-v|<\delta\} \subset \subset D_{r, \beta}$ and the boundary of this disc meets $D_{r, \beta}$ in a point $u_{1}$. By the definition of $v$, we see that $\delta \leqslant \varepsilon / 2$, which implies $\left|u_{1}-w\right|<\varepsilon$. But $u_{1} \in E$ and, hence $E$ is everywhere dense in $\partial D_{r, \beta}$.

Choose an arbitrary $u_{1} \in E$ and let $v$ be the corresponding point in $C D_{r, \beta}$. Then $f(\zeta) / \zeta \neq v$ for all $f \in K_{\beta}$ and $\log (f(\zeta) / \zeta-v)$ is analytic on $\{(f(\zeta), \zeta)$ : $\left.f \in K_{\beta}\right\}$. Applying Theorem 2.1 in the case $0<\beta<1$ we find that

$$
m=\min _{f \in K_{\beta}} \log \left|\frac{f(z)}{z}-v\right|=\min _{f \in K_{\beta}} \operatorname{Re} \log \left(\frac{f(z)}{z}-v\right)
$$

is attained only for a function of the form

$$
F_{x, y}(z)=\frac{1}{\beta+1} \frac{1}{x-y}\left[\left(\frac{1+x z}{1+y z}\right)^{\beta+1}-1\right], \quad x \neq y,|x|=|y|=1 .
$$

By construction, $m=\log \left|u_{1}-v\right|$ so that every point of $E$ corresponds to some $F_{x, y}$. If $u \in \partial D_{r, \beta}$ is arbitrary, there is a sequence $\left\{u_{n}\right\} \subset E$ with $\lim _{n \rightarrow \infty} u_{n}=u$. For some $x_{n} \neq y_{n}$ of modulus $1, u_{n}=F_{x_{n}, y_{n}}(\zeta) / \zeta$, and a subsequence of $\left\{F_{x_{n}, y_{n}}\right\}$ converges to a function again of the form $F_{x, y}$, where here it is possible that $x=y$. Hence

$$
u=\frac{F_{x, y}(\zeta)}{\zeta}=\frac{1}{\beta+1} \frac{1}{(x-y) \zeta}\left[\left(\frac{1+x \zeta}{1+y \zeta}\right)^{\beta+1}-1\right] .
$$

If we set $x \zeta=s$ and $y \zeta=t$, we obtain the desired result.

(b) Set

$$
f_{\beta}(s, t)=\frac{1}{\beta+1} \frac{1}{s-t}\left[\left(\frac{1+s}{1+t}\right)^{\beta+1}-1\right] .
$$

We first show

$$
D_{r, \beta} \supset\left\{f_{\beta}(s, t):|t| \leqslant|s|=r \text { or }|s| \leqslant|t|=r\right\} .
$$

To do this we fix $t,|t|=r$, and consider the curve $\Gamma_{\beta}$ defined by

$$
\Gamma_{\beta}(s)=f_{\beta}(s, t)=\frac{(1+s)^{\beta+1}-(1+t)^{\beta+1}}{(\beta+1)(s-t)(1+t)^{\beta+1}}, \quad|s|=r .
$$

Then $\Gamma_{\beta}(t)=(1+t)^{-1}$ and $\Gamma_{0}(s)=(1+t)^{-1}$, i.e., $\Gamma_{0}$ reduces to a single point. 
Suppose $z \in \operatorname{int} \Gamma_{\beta}$ (interior of $\Gamma_{\beta}$ ), i.e., the index of $z$ with respect to $\Gamma_{\beta}, n\left(\Gamma_{\beta}, z\right)$, is nonzero. We claim $z \in \Gamma_{\alpha}$ for some $\alpha, 0<\alpha<\beta$. Otherwise, consider the function $H:\{|s|=r\} \times[0, \beta] \rightarrow \mathrm{C}-\{z\}$ defined by $H(s, \beta)$ $=\Gamma_{\beta}(s) . H$ is a homotopy of $\Gamma_{0}$ to $\Gamma_{\beta}$ relative to $\left\{(1+t)^{-1}\right\}$ in $\mathbf{C} \backslash\{z\}$. This would imply

$$
n\left(\Gamma_{\beta}, z\right)=\frac{1}{2 \pi i} \int_{\Gamma_{\beta}} \frac{d w}{w-z}=\frac{1}{2 \pi i} \int_{\Gamma_{0}} \frac{d w}{w-z}=n\left(\Gamma_{0}, z\right)=0,
$$

contradicting the assumption made on $z$. Consequently every point $z \in \operatorname{int} \Gamma_{\beta}$ corresponds to an extremal function in $K_{\alpha}$ for some $\alpha, 0<\alpha<\beta$. Since $K_{\alpha} \subset K_{\beta}$, we conclude that int $\Gamma_{\beta} \subset D_{r, \beta}$ and by the argument principle we obtain $\left\{f_{\beta}(s, t):|s| \leqslant|t|=r\right\} \subset D_{r, \beta}$. Interchanging the roles of $s$ and $t$, the above argument shows that $\left\{f_{\beta}(s, t):|t| \leqslant|s|=r\right\} \subset D_{r, \beta}$, which gives us (3.1). Suppose $0<r_{1}<r<1$ and $u=f(\xi) / \xi \in D_{\eta, \beta},|\xi|=r_{1}$. If we define $g(z)=f(\tau z) / \tau$ where $\tau=r_{1} / r$, then $g \in K_{\beta}$. Set $\zeta=\xi / \tau$, so that $|\zeta|=r$ and, consequently,

$$
u=f(\xi) / \xi=g(\zeta) / \zeta \in D_{r, \beta},
$$

which shows that $D_{r_{1}, \beta} \subset D_{r, \beta}$. We note that $D_{0, \beta}=\{1\}$. Hence

$$
\begin{aligned}
D_{r, \beta} & =\underset{0<r_{1}<r}{\bigcup} D_{r_{1}, \beta} \\
& \supset \underset{0<r_{1} \leqslant r}{\bigcup}\left\{f_{\beta}(s, t):|s| \leqslant|t|=r_{1} \text { or }|t| \leqslant|s|=r_{1}\right\} \\
& =\left\{f_{\beta}(s, t):|s|,|t| \leqslant r\right\} .
\end{aligned}
$$

Let $C_{r, \beta}$ denote $\left\{f_{\beta}(s, t):|s|,|t| \leqslant r\right\}$. For $w \neq 1$ and $w \in D_{r, \beta}$, set $r_{0}$ $=\inf \left\{x: w \in D_{x, \beta}\right\}$. Clearly $w \in D_{r_{0}, \beta}$. For $f \in K_{\beta}$, let $\hat{f}$ denote the analytic function defined by $\hat{f}(z)=f(z) / z$. The set $\cup_{x<r_{0}} D_{x, \beta}$ is open since it equals

$$
\bigcup_{x<r_{0}}\left\{\hat{f}(\zeta):|\zeta|=x, f \in K_{\beta}\right\}=\bigcup_{f \in K_{\beta}}\left\{\hat{f}(\zeta):|\zeta|<r_{0}\right\}
$$

We then have

$$
\begin{aligned}
D_{r_{0}, \beta} & =\bigcup_{x \in r_{0}} D_{x, \beta}=\bigcup_{f \in K_{\beta}} \hat{f}\left(|z| \leqslant r_{0}\right)=\bigcup_{f \in K_{\beta}} \overline{\hat{f}\left(|z|<r_{0}\right)} \\
& \subset \overline{f \in K_{\beta}} \hat{f\left(|z|<r_{0}\right)}=\overline{x<r_{0}} D_{x, \beta} \subset \overline{D_{r_{0}, \beta}}=D_{r_{0}, \beta},
\end{aligned}
$$

showing that $D_{r_{0}, \beta}$ is the closure of the open set $\cup_{x<r_{0}} D_{x, \beta}$. If $V$ is an arbitrary disc about $w, V$ meets $D_{x, \beta}$ for some $x$ sufficiently near and less than $r_{0}$. Since $w \in \mathcal{C} D_{x, \beta}, V$ contains a point of $\partial D_{x, \beta}$. Hence there exists a sequence 
$w_{n} \in \partial D_{x_{n}, \beta}$ whereby $x_{n} \rightarrow r_{0}$ and $w_{n} \rightarrow w$. But by part (a) of the theorem, $w_{n}=f_{\beta}\left(s_{n}, t_{n}\right)$ where $\left|s_{n}\right|=\left|t_{n}\right|=x_{n}$ implying that $w=f_{\beta}(s, t)$ where $|s|$ $=|t|=r_{0}$. Therefore $w \in C_{r_{0}, \beta} \subset C_{r, \beta}$, i.e., $D_{r, \beta} \subset C_{r, \beta}$, which completes the proof.

As a second application of Theorem 1.2 we consider the problem of determining the radius of starlikeness, $r_{s}(\beta)$, for the class $K_{\beta}$. The case $\beta=1$ was solved by Lewandowski [9], who showed that $r_{s}(1)=4 \sqrt{2}-5$.

Corollary 3.2. If $0<\beta \leqslant 1$,

$$
r_{s}(\beta)=\min \left\{r: \operatorname{Re} f_{\beta}(s, t)=0 \text { on }|s|=|t|=r\right\} .
$$

Proof. The function

$$
g(z)=\frac{f\left((z+\zeta) /\left(1+\bar{\zeta}_{z}\right)\right)-f(\zeta)}{f^{\prime}(\zeta)\left(1-|\zeta|^{2}\right)} \in K_{\beta}
$$

if $f \in K_{\beta}$ and $|\xi|<1$. Setting $z=-\zeta$, we obtain

$$
\frac{g(-\zeta)}{-\zeta}=\frac{1}{1-|\zeta|^{2}} \frac{f(\zeta)}{\zeta f^{\prime}(\zeta)}
$$

The function $f$ is starlike in $|\zeta|<r$ if and only if $\operatorname{Re}\left\{\zeta f^{\prime}(\zeta) / f(\zeta)\right\}>0$ for $|\zeta|<r$. Hence $r_{s}(\beta)$ is the largest value of $r, 0<r<1$, such that $\operatorname{Re}\left\{\zeta f^{\prime}(\zeta) / f(\zeta)\right\} \geqslant 0$ for every $f \in K_{\beta}$. By (3.2), $r_{s}(\beta)$ is also the largest value of $r$ satisfying $\operatorname{Re}\{f(\zeta) / \zeta\} \geqslant 0$ and this is the smallest value of $r$ such that $D_{r, \beta}$ cuts the imaginary axis, which must then occur at some point of $\partial D_{r, \beta}$. The proof is completed by applying Theorem 3.1(a).

\section{BIBLIOGRAPHY}

1. R. W. Barnard, Extremal problems for univalent functions whose ranges contain a fixed disc, Ph. D. Dissertation, Univ. of Maryland, 1971.

2. M. Biernacki, Sur la représentation conforme des domaines linéairement accessibles, Prace Mat.-Fiz. 44 (1936), 293-314.

3. D. A. Brannan, J. G. Clunie and W. E. Kirwan, On the coefficient problem for functions of bounded boundary rotation, Ann. Acad. Sci. Fenn. Ser. AI No. 523 (1973), 18 pp.

4. J. A. Hummel, Extremal problems in the class of starlike functions, Proc. Amer. Math. Soc. 11 (1960), 741-749. MR 22 \#11133.

5. G. Julia, Sur une equation aux dérivées fonctionelles liée da la représentation conforme, Ann. Sci. École Norm. Sup. 39 (1922), 1-28.

6. W. K. Kaplan, Close-to-convex schlicht functions, Michigan Math. J. 1 (1952), 169-185 (1953). MR 14, 966.

7. W. E. Kirwan, A note on extremal problems for certain classes of analytic functions, Proc. Amer. Math. Soc. 17 (1966), 1028-1030. MR 34 \#2854.

8. - Extremal problems for functions with bounded boundary rotation, Ann. Acad. Sci. Fenn. No. 595 (1975), 19 pp. 
9. Z. Lewandowski, Sur l'identité de certaines classes de fonctions univalentes. II, Ann. Univ. Mariae Curie-Skłodowska Sect. A 14 (1960), 19-46. MR 28 \#200.

10. V. Paatero, Uber die konforme Abbildung von Gebieten, deren Rander von beschränkter Drehung sind, Ann. Acad. Sci. Fenn. Ser. A 33 (1931), 1-79.

11. Ch. Pommerenke, On close-to-convex analytic functions, Trans. Amer. Math. Soc. 114 (1965), 176-186. MR 30 \#4920.

12. M. S. Robertson, Variational methods for functions with positive real part, Trans. Amer. Math. Soc. 102 (1962), 82-93. MR 24 \# A3288.

13. M. M. Schiffer and O. Tammi, A method of variations for functions with bounded boundary rotation, J. Analyse Math. 17 (1966), 109-144. MR 35 \#5601.

14. E. Strohhäcker, Beiträge zur Theorie der schlichten Funktionen, Math. Z. 37 (1933), 356-380.

Department of Mathematics, Federal City College, Washington, D. C. 20001 